\title{
Process-oriented aggregation scorecards operational control forwarding activities
}

\section{Krupensky Nikita Aleksandrovich}

Russian Federation, Ph. D., Senior Researcher, Business Center "Research on transportation problems of megacities".

Higher School of Economics - National Research University, 101000, Russian Federation, Moscow, Str. Myasnitskaya, 20. Tel.: +7 (495) 771-32-32. http://www.hse.ru

\section{krupensky@yandex.ru}

\section{Ivakhnenko Andrey Mikhailovich}

Russian Federation, Doctor of Technical Sciences, Professor, Header of Department of «Management».

State Technical University - MADI, 125319, Russian Federation, Moscow, Leningradsky prospekt, 64. Tel.: +7 (499) 151-64-12. http://www.madi.ru

\section{jointlab@mail.ru}

\section{Ostroukh Andrey Vladimirovich}

Russian Federation, full member RAE, Doctor of Technical Sciences, Professor, Department of «Automated Control Systems».

State Technical University - MADI, 125319, Russian Federation, Moscow, Leningradsky prospekt, 64. Tel.: +7 (499) 151-64-12. http://www.madi.ru

\section{ostroukh@mail.ru}

\begin{abstract}
The paper proposed and substantiated scientific approach to development of a unified control system for freight forwarding activities (FD) on the basis of applied information systems to improve the efficiency and manageability of freight forwarding company.
\end{abstract}

A mathematical model for calculating targets for process control in forwarding activities, as well as the method of formation of the system of control procedures and control algorithm and eliminate incidents, integrated information system to support management decisions (DSS ).

Theoretical basis of the study are the works of Russian and foreign authors in the field of the modern theory of the development of transport, as well as control systems for DSS. Formation control procedures carried out on the basis of comparison of the main tasks and milestones of the parameters on which the performance evaluation, and its generalization in the system was 
based on the deductive method. Research methodology uses a systematic approach to identify the features of the system as a whole depending on the effect of changes in each of the elements that compose it. When developing formal models of components used methods of general systems theory, the classical set-theoretic apparatus. System analysis activities conducted on the basis of real statistical data processed using the methods of experimental design.

The proposed control system to determine the optimal parameters of the transport chain, taking into account the specifics of each transaction and the specific conditions of carriage in close connection with the parameters of the environment and management objectives of the enterprise.

Set of scientific principles, ideas and practical research results, developed methods and algorithms have been tested and implemented for practical application in The Association of International Road Transport Carriers (ASMAP) and company BDP International.

Keywords: forwarding activities, road transportation, information systems, decision support systems, operational control, process approach. 
ISSN 2306-1561

Автоматизация и управление в технических системах (АУТС) 2014. - №1.2(9). - C. 123-142.

DOI: $10.12731 / 2306-1561-2014-1-26$

УДК 004.9: 656.025

\section{Процессно-ориентированное агрегирование системы показателей оперативного контроля транспортно-экспедиционной деятельности}

\section{Крупенский Никита Александрович}

Российская Федерация, кандидат технических наук, старший научный сотрудник Центра исследования транспортных проблем мегаполисов.

ФГБОУ ВПО «Высшая школа экономик», 101000, Российская Федерация, г. Москва, ул. Мясницкая, д. 20, Тел.: +7 (495) 771-32-32, http://www.hse.ru

krupensky@yandex.ru

\section{Ивахненко Андрей Михайлович}

Российская Федерация, доктор технических наук, профессор, заведующий кафедрой «Менеджмент».

ФГБОУ ВПО «Московский автомобильно-дорожный государственный технический университет (МАДИ)», 125319, Российская Федерация, г. Москва, Ленинградский проспект, д.64, Тел.: +7 (499) 151-64-12, http://www.madi.ru

jointlab@mail.ru

\section{Остроух Андрей Владимирович}

Российская Федерация, академик РАЕ, доктор технических наук, профессор кафедры «Автоматизированные системы управления».

ФГБОУ ВПО «Московский автомобильно-дорожный государственный технический университет (МАДИ)», 125319, Российская Федерация, г. Москва, Ленинградский проспект, д.64, Тел.: +7 (499) 151-64-12, http://www.madi.ru

ostroukh@mail.ru

Аннотация. В статье предложен и обоснован научный подход к разработке унифицированной системы контроля в транспортно-экспедиционной деятельности (ТЭД) на основе применяемых информационных систем для повышения эффективности и управляемости транспортно-экспедиционного предприятия.

Разработана математическая модель расчета плановых показателей для целей управления процессами в транспортно-экспедиционной деятельности, a также методика формирования системы контрольных процедур и алгоритм контроля и 
устранения инцидентов, интегрированные в информационную систему поддержки принятия управленческих решений (СППР).

Теоретической основой исследования являются труды отечественных и зарубежных авторов в области современной теории развитии транспорта, а также систем контроля в СППР. Формирование контрольных процедур осуществлялось на основе метода сравнения основных задач, этапов реализации и параметров, по которым производится оценка эффективности, а их обобщение в систему проводилось на основе дедуктивного метода. Методология исследования использует системный подход с целью выявления особенностей функционирования системы в целом в зависимости от влияния изменений, происходящих в каждом из элементов, ее образующих. При разработке формальных моделей компонентов использовались методы общей теории систем, классический теоретико-множественный аппарат. Системный анализ деятельности проводился на базе реальных статистических данных, обработанных с использованием методов планирования эксперимента.

Предложенная система контроля позволяет определить оптимальные параметры транспортной цепи с учетом специфики каждой сделки и конкретных условий перевозки в тесной связи с параметрами внешней среды и управленческими задачами предприятия.

Совокупность научных положений, идей и практических результатов исследований, разработанные методы и алгоритмы прошли апробацию и внедрены для практического применения в Ассоциации Международных Автомобильных Перевозчиков (АСМАП) и компании BDP International.

Ключевые слова: транспортно-экспедиционная деятельность, автомобильные перевозки, информационные системы, системы поддержки принятия решений, оперативный контроль, процессный подход.

\section{1. Введение}

Главным критерием выживания транспортно-экспедиционных предприятий (ТЭП) является поддержание высокой конкурентоспособности, что невозможно без поиска новых возможностей для бизнеса. Продвижение бизнеса в условиях большой неопределенности определяет оперативный характер деятельности предприятия. Именно на этом этапе возникают существенные искажения информации и наибольшее число рисков, связанных со сбоями в осуществлении управленческих задач.

Одним из препятствий на пути развития транспортно-экспедиционных предприятий становится качество управления. При этом ключевым процессом является контроль, реализация которого позволяет обеспечить обратную связь и принимать оптимальные управленческие решения. Обеспечение системы организации контроля в условиях процессного управления особенно актуально, так как дает возможность получить в относительно короткий срок достоверную и качественную информацию и в транспортной цепи и по регулированию ресурсной нагрузки. 
Существующие методологические подходы к исследованию проблемы обеспечения устойчивой деятельности ТЭП развивают отдельные аспекты, однако многоаспектность этой проблемы заставляет по-новому рассмотреть принципы построения контрольных управленческих функций, которые сегодня не раскрывают взаимосвязь с процессом управления и оценкой эффективности деятельности предприятия, что приводит к снижению роли управления. Именно этим обуславливается необходимость научного обоснования повышения значимости системы контроля в транспортно-экспедиционной деятельности.

\section{2. Современные тенденции развития и особенности управления транспортно-экспедиционным предприятием}

Транспортно-экспедиционная деятельность представляет совокупность организационных и технологических взаимосвязанных действий и операций, выполняемых ТЭП и их подразделениями самостоятельно или согласованно с другими организациями при подготовке, осуществлении и завершении перевозок грузов. Структура транспортно-экспедиционной деятельности включает:

- управление движением транспортных средств;

- координацию работы разных видов транспорта;

- выбор типа и определение необходимого количества подвижного состава для перевозок;

- нормирование скоростей движения транспорта;

- определение сферы целесообразности использования разных видов транспорта в зависимости от конкретных условий перевозок, вида и свойств грузов, эксплуатационных показателей транспорта;

- обеспечение эффективных и безопасных перевозок грузов;

- оперативный контроль процесса перевозки;

- применение экономико-математических методов и расчетов для повышения эффективности и снижения затрат на перевозки;

- разработку грузопотоков на основе материалов обследований: рациональных маршрутных схем, предусматривающих изменение направлений существующих маршрутов и открытие новых.

Значительное количество работ исследователей сосредоточено на рассмотрении вопросов по совершенствованию системы организации и управления деятельностью транспортно-экспедиционного предприятия в условиях рынка, а также вопросам управления и контроля в целях принятия оптимальных управленческих решений [1 22].

В работах $[1-6,10,11,13$ - 15] даны конкретные рекомендации по оптимизации функционирования и взаимодействия различных видов транспорта и организации грузовых перевозок в международных сообщениях.

Исследования информационного обеспечения транспортно-экспедиционной деятельности, в том числе, разработка алгоритмов автоматизированного 
проектирования информационных процессов для повышения эффективности управления достаточно глубоко отражены в работах $[1-6,10,11]$.

В основном рассматривается стандартная организационная система управления транспортно-экспедиционным предприятием при осуществлении текущей деятельности. Вопрос управления транспортно-экспедиционным предприятием при осуществлении оперативной деятельности практически не поднимается. Недостаточно изучен вопрос применения действующих ИС для обеспечения поддержки принятия оперативных управленческих решений. Кроме того при увеличивающемся объеме информации остаются недостаточно проработанными вопросы применения в транспортно-экспедиционной деятельности методов контроля используемых в других видах деятельности.

Скоординированное объединение и выполнение процессов менеджмента транспортных услуг естественным образом обеспечивает более эффективное управление. Методология менеджмента качества, известная как "Планируй - Выполняй - Контролируй - Действуй", может применяться практически ко всем процессам транспортно-экспедиционной деятельности:

- планируй - установите цели и процессы для получения результатов, соответствующих требованиям заказчика;

- выполняй - осуществляйте эти процессы;

- контролируй - проводите мониторинг и измеряйте процессы и услуги по отношению к политике, целям и требованиям;

- действуй - предпринимайте действия для постоянного совершенствования характеристик процессов.

Ключевым процессом является процесс контроля, цель которого в измерении и ревизии для подтверждения того, что цели менеджмента услуг достигнуты и план выполнен. Цели проведения ревизии менеджмента услуг должны быть зарегистрированы, после чего определены необходимые корректирующие действия. Информация о существенных несоответствиях должна быть доведена до сведения заинтересованных сторон. В целях повышения эффективности транспортных услуг все предложенные усовершенствования должны быть ранжированы по приоритетам и закреплены за ответственными исполнителями.

Существующие решения по автоматизации [8 - 15] могут либо решать множество задач ТЭП, в том числе анализировать расхождения между плановыми и фактическими показателями во временных, количественных и финансовых измерителях, но быть дорогими и «тяжелыми», как SAP и поэтому не подходить для малого и среднего бизнеса, либо быть достаточно дешевыми и удобными, осуществляя непрерывный мониторинг процессов и учетные функции и не специализироваться на анализе процессов в режиме реального времени. Часть программных продуктов требует сложных дополнительных разработок в целях адаптации системы для нужд организации, а часть применяется только для организации деятельности одного вида перевозок. Информационная система (ИС) должна не просто формировать отчеты, содержащие оценку достигнутых результатов, но и обнаруживать исключительные 
ситуации, требующие особого внимания. Возникает необходимость использовать новые возможности управления предприятием за счет внедрения подсистемы контроля в информационных системах.

\section{3. Формализация системы управления и контроля транспортно- экспедиционной деятельностью}

Схему управления и контроля транспортно-экспедиционной деятельностью можно представить в виде «петли управления» $[10,11]$, включающей циклическую последовательность следующих этапов: прогноз - планирование - деятельность по реализации планов - учет и анализ результатов - коррекция прогнозов и планов.

Ключевую роль в системе управления и контроля играет информационное обеспечение. Для осуществления контрольных функций необходимо, чтобы информационный поток опережал материальный поток или соответствовал ему. Для оценки эффективности управления транспортных потоков предлагается выбор ключевых показателей на основе ключевых целей.

При постановке задачи расчета плановых показателей для определения их величины, исходя из прогнозируемого спроса на услуги перевозки, возникает необходимость в разработке математической модели. Для построения математической модели рассматривается логическая схема взаимодействия (рисунок 1).

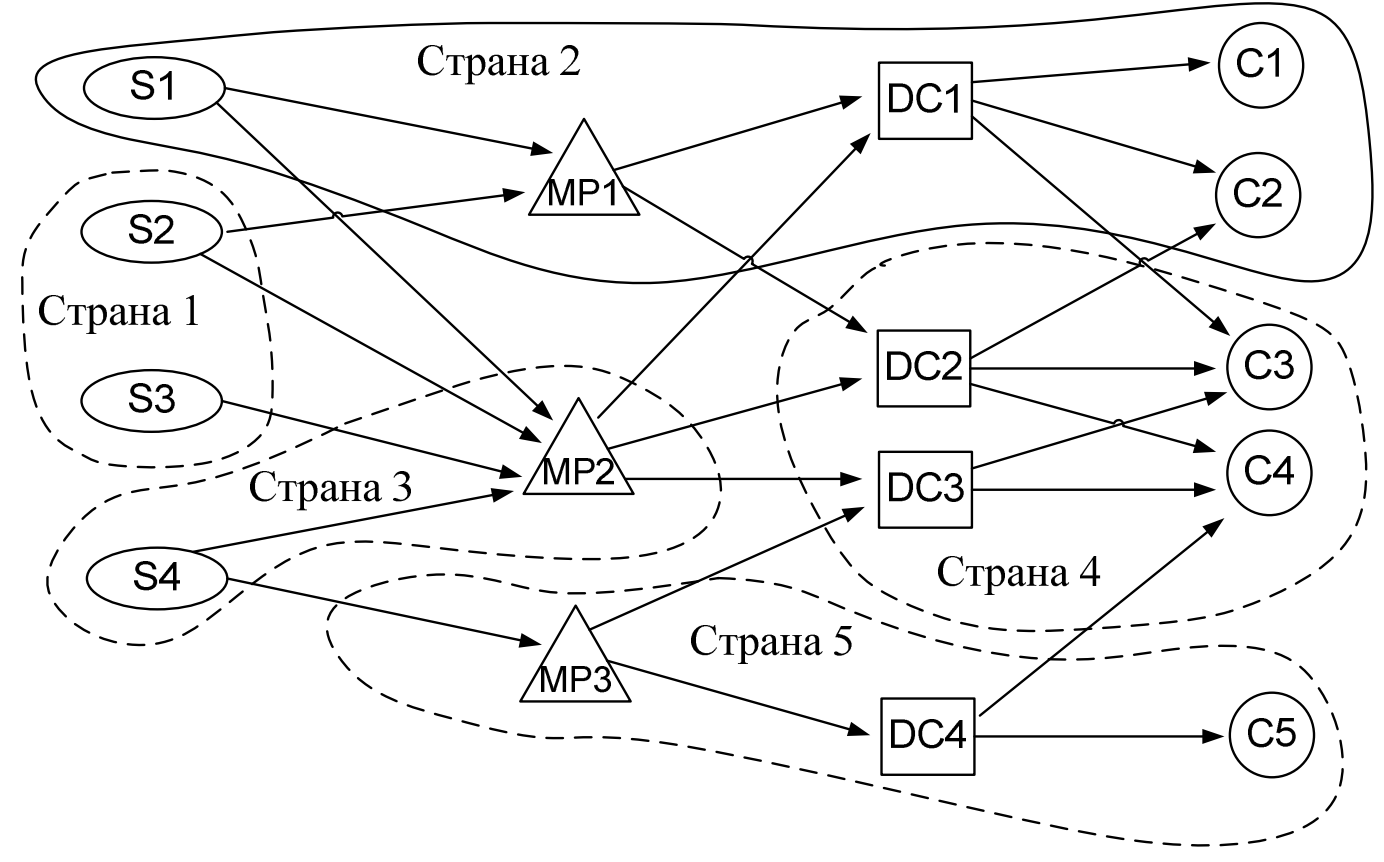

Рисунок 1 - Логическая схема взаимодействия

В модель вводятся следующие параметры: $c_{i}-$ стоимость фрахта на $i$-й период, $E_{i}$ - стоимость заказа на $i$-й период, $h_{i}$ - удельные затраты в течении рассматриваемого $i$ го периода, $u_{i}$ - удельные потери $i$-го периода, $r_{i}$ - удельный доход $i$-го периода, $\alpha_{i}-$ 
коэффициент дисконтирования на $i$-й период, $D_{i}$ - величина случайного заказа на $i$-й период, $f\left(D_{i}\right)$ - плотность вероятности $D_{i}$ на $i$-й период.

Рассматривается $m n$ заказов, где $n$ - число периодов. Пусть $F_{i}\left(x_{i}\right)$ - максимальная суммарная ожидаемая прибыль для заказов от $i$ до $m n$, где $x_{i}$ - транспортный резерв перед размещением $i$-го заказа. При реализации данной задачи будем использовать принцип оптимальности Беллмана. Состояние системы в виде заказа $y_{i}$ единиц транспорта зависит от стоимости фрахта, стоимости заказа, удельных затрат и др. Управлением на каждом шаге является выбор: делать ли заказ и на какое количество единиц транспорта или не делать его вовсе.

Первые два выборочных момента количества возможных заказов являются несмещенными оценками для среднего значения и дисперсии соответственно и являются двумя первыми $k$-статистиками Фишера, где $k_{1}$ и $k_{2} n$-мерные векторы

$k_{1}=\int_{-\infty}^{\infty}(D f(D)) d D, k_{2}=\int_{-\infty}^{\infty}\left(D^{2} f(D)\right) d D$.

В данной задаче рассматриваются n выборок из нормальной совокупности, т.е. из $N\left(k_{11}, k_{21}\right), \ldots, N\left(k_{1 n}, k_{2 n}\right)$. При этом

$N\left(\sum_{i=1}^{n} b_{i} k_{1 i}, \sum_{i=1}^{n} \frac{b_{i}^{2} k_{2 i}}{m_{i}}\right)$,

где bi - некоторые константы.

Запишем поставленную задачу в виде задачи динамического программирования: $F_{i}\left(x_{i}\right)=\max _{y_{i} \geq x_{i}}\left\{-c\left(y_{i}-x_{i}\right)+\int_{0}^{y_{i}}\left(r D-h\left(y_{i}-D\right)\right) f(D) d D+\int_{y_{i}}^{\infty}\left(r y_{i}-\alpha r\left(y_{i}-D\right)+\right.\right.$ $\left.\left.u\left(y_{i}-D\right)\right) f(D) d D+\alpha \int_{0_{i}}^{\infty} F\left(y_{i}-D\right) f(D) d D\right\}$.

Так как функция ожидаемой прибыли $\mathrm{Fi}(\mathrm{xi})$ является вогнутой, то оптимальное решение можно определить из условия, в котором уравнение записано в векторном виде (из $\left.R_{n}\right)$ :

$\frac{\partial F}{\partial y}=-c-h \int_{0}^{y} f(D) d D+\int_{y}^{\infty}((1-\alpha) r+u) f(D) d D+\alpha \int_{0}^{\infty}\left(\frac{\partial F(y-D)}{\partial y}\right) f(D) d D=0$,

где величина $\left(\frac{\partial \mathrm{F}(\mathrm{y}-\mathrm{D})}{\partial \mathrm{y}}\right)=$ с определяется следующим образом: если на начало следующего этапа еще имеются заказы, то прибыль возрастет на величину им пропорциональную, а объем следующих заказов уменьшается на эту величину

$\left.-c-h \int_{0}^{y} f(D) d D+((1-\alpha) r+u)\left(1-\int_{0}^{y} f(D) d D\right)+\alpha c \int_{0}^{\infty} f(D) d D=0\right)$

Поэтому оптимальный уровень заказа у* определяется из уравнения

$\int_{0}^{y^{*}} f(D) d D=\frac{u+(1-\alpha)(r-c)}{u+h+(1-\alpha) r}$.

Оптимальная стратегия каждого этапа при наличии хі единиц резерва выражается следующим правилом: если $x_{i}<y_{i}^{*}$, то брать заказ на величину $z_{i}=y_{i}^{*}-x_{i}$ единиц, иначе заказ не брать $\left(z_{i}=0\right)$. Соответствующая функция производственных затрат на $i$-том этапе имеет вид

$C_{i}\left(z_{i}\right)=\left\{\begin{array}{c}0, z_{i}=0 \\ E_{i}+c_{i}\left(z_{i}\right), \quad z_{i}>0\end{array}\right.$ 
Основная цель состоит в том, чтобы с помощью выборочного метода получить информацию, на основании которой можно высказать некоторые утверждения или сделать выводы относительно $F(x)$ или некоторых ее свойств. Для его построения достаточно ограниченности моментов первого и второго порядков k1, k2.

Рассмотрим выборку $D=\left(D_{1}, D_{2}, \ldots, D_{n}\right)$ с распределением $N\left(k_{1}, k_{2}\right)$ и получим ее интервальные оценки. Так как величина $\frac{\sqrt{n}\left(\bar{D}-k_{1}\right)}{\sqrt{k_{2}}}$ на отрезке $\left[t_{1}, t_{2}\right]$ имеет распределение Стьюдента, то

$P\left\{t_{1}<\frac{\sqrt{n}\left(\bar{D}-k_{1}\right)}{\sqrt{k_{2}}}<t_{2}\right\}=\gamma$.

Таким образом, $\left(\bar{D}-t_{2} \sqrt{\frac{k_{2}}{n}}, \bar{D}-t_{1} \sqrt{\frac{k_{1}}{n}}\right)$ является наблюдаемым случайным доверительным интервалом $\mathrm{c}$ вероятностью $\gamma$. Чаще всего рассматривается доверительный интервал с $\gamma=0,95$. В регрессионном анализе при оценке результатов планирования их средние значения задаются функцией регрессии $G\left(y_{i}\right)=\sum_{j=1}^{n} \beta_{j} x_{j i}$. В результате оценки параметров $\beta \mathrm{i}$, учитывая, что $\mathrm{G}^{-1}\left(\beta_{\mathrm{j}}\right)=\sum_{\mathrm{i}=1}^{\mathrm{n}} \alpha_{\mathrm{ij}} \mathrm{y}_{\mathrm{i}}$, получаем $G\left(G^{-1}\left(\beta_{j}\right)\right)=\sum_{j=1}^{n} \sum_{i=1}^{n} \beta_{j} \alpha_{i j} y_{i}$ откуда ясно, что аіј должны удовлетворять условию $\sum_{r=1}^{n} \alpha_{j r} x_{i r}=\delta_{i j}$, где $\delta_{i j}$ - символ Кронекера. Следовательно, согласно теореме Гаусса-Маркова оценка с минимальной дисперсией для $\beta$ рі равна

$\beta_{j}=\sum_{i=1}^{n}\left(\sum_{r=1}^{n} x_{i r} x_{j r} \sum_{r=1}^{n} x_{i r} y_{r}\right)$.

В системах, базирующихся на обмене информацией, целесообразно выделять два типа организационных элементов: включающие и не включающие основную деятельность. Элементы первого типа являются потребителями-поставщиками (конечными) информации и могут взаимодействовать как непосредственно (реализуя информационную деятельность в собственных организационных рамках), так и посредством элементов второго типа, которые представляют собой промежуточных потребителей- поставщиков информации, или информационные системы.

Общее представление о взаимодействии пользователей системы информационного обмена представлено на рисунке 2.

В данном представлении уровни взаимодействия разделены на 3 типа:

- непосредственное рабочее взаимодействие (связь 3-3) представляет собой постоянный обмен информацией в группе или коллективе в процессе совместной деятельности;

- непосредственное документальное взаимодействие (связь 4-2) заключается в оформлении результата и ограниченном контролируемом распространении (например - передача отчета или документации заказчику);

- опосредованное документальное взаимодействие (связь 5-1) состоит в опубликовании результата и его последующем неограниченном перемещении по каналам ИС. 
- Управление информационным обменом (информационными ресурсами) на макроуровне может быть разделено на:

- организацию работ и взаимодействия агентов и соисполнителей при выполнении технологических процессов транспортно-экспедиционной деятельности (связи 3-3);

- маркетинг - поиск заказчиков, получение заказов, связь с заказчиками, оформление и передача результатов, поиск прочих возможных потребителей результатов (связи 4-2);

- распространение информации в документальной форме по каналам ИС, решение задач повышения полноты, точности, оперативности информационного обмена и обслуживания (связи 5-1).

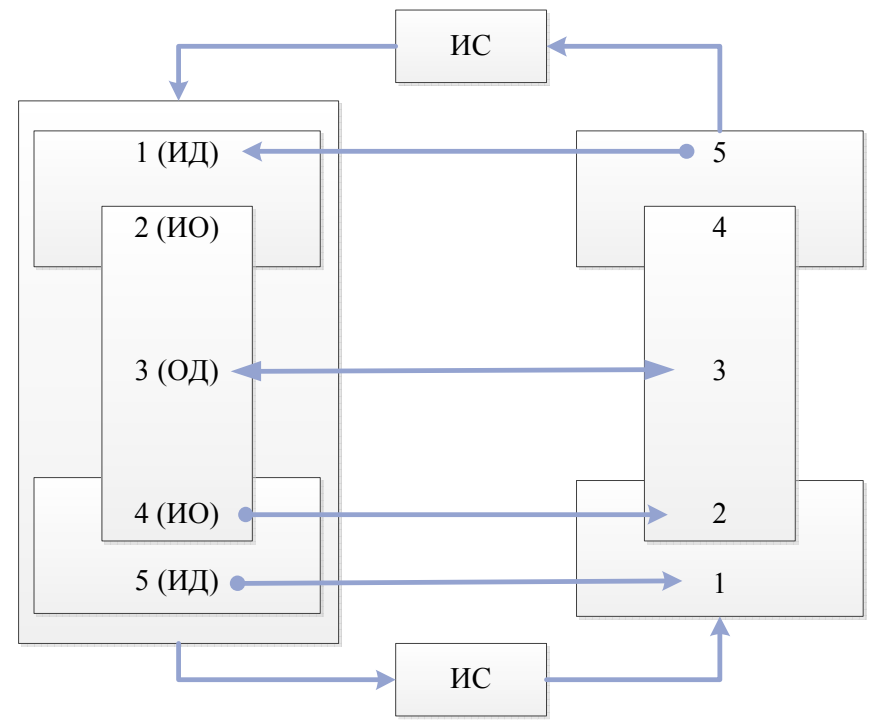

\section{ИД - информационная деятельность; ИО - информационный обмен; ОД - основная деятельность.}

\section{Рисунок 2 - Уровни взаимодействия потребителей-поставщиков информации}

Далее в работе выполнена реализация метода выбора управленческого решения в информационном графе, алгоритме для общей задачи управления перевозками. Понятие информационного графа (ИГ) над базовым множеством $I G=\langle W, V\rangle$ определяется следующим образом (рисунок 3).

Рассматривается конечная многополюсная ориентированная сеть. На диаграммах она изображается в виде точек-полюсов, некоторые из которых соединены линиями. Выбирается корневой полюс. Остальные полюса являются листьями и им приписываются записи из некоторого множества Z, причем разным листьям могут быть приписаны одинаковые записи. Некоторые вершины сети (в том числе и полюса) являются переключательными, и им приписываются переключатели из $V=\left\{v_{i}, i \in I\right\}$. Ребра, исходящие из каждой переключательной вершины, нумеруются подряд, начиная с 1, и представляют переключательные ребра. Ребра, не являющиеся 
переключательными, являются предикатными и им приписываются предикаты из множества $W=\left\{w_{j}, j \in J\right\}$.

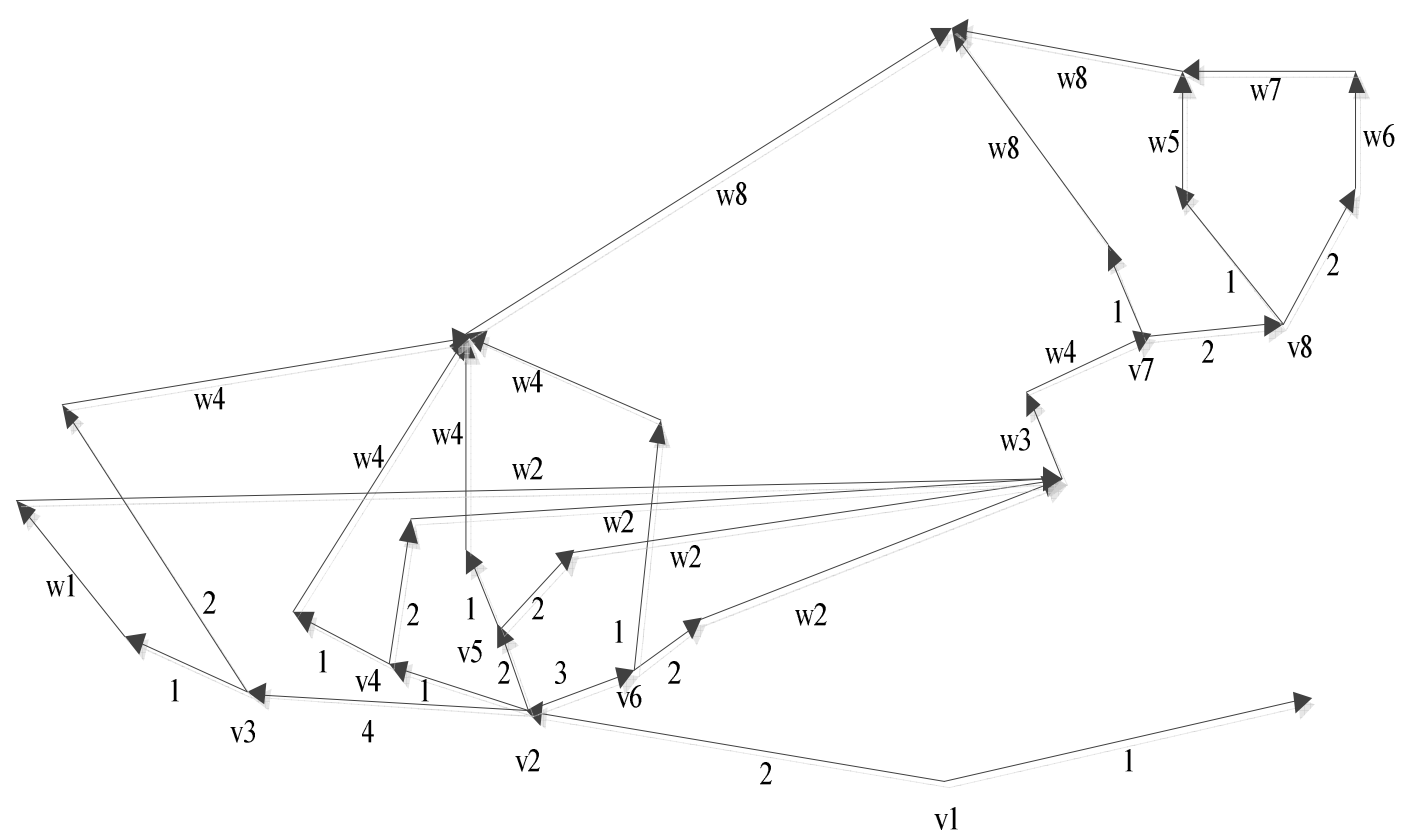

Рисунок 3 - Информационный граф системы контроля

Нагруженная многополюсная ориентированная сеть над базовым множеством $I G$ $=\langle W, V\rangle$ представляет информационный граф (ИГ). При этом $V=\left\{v_{i}, i \in I\right\}, W=$ $\left\{w_{j}, j \in J\right\}, I, J-$ конечные множества индексов. Алгоритмы блок-схемы, приведенные в данной работе, реализуются с помощью ИГ.

Для целей построения и реализации информационного графа введено понятие задачи информационного поиска (ЗИП). В работе ЗИП осуществляется следующим образом.

1) На основе имеющейся информации, полученной от системы контрольных процедур, описанных выше, такой, например, как «Проверка сроков заключения договора», «Расчет рисков», «Проверка цены заказа» и др., формируется библиотека (множество записей для поиска по ключу).

2) Заказчиком создается ключ-запрос для поиска, то есть составляется список пожеланий относительно будущего заказа.

3) Управление выбором заказа передается модулю-алгоритму, то есть реализуется описанная математическая модель, которая выдает окончательный итог в виде рекомендации по заключению контракта для данного заказа.

ЗИП в работе представляет тройку $S_{z}=(X, Y, p)$, где $X$ - множество запросов, $Z$ подмножество множества $Y$ (или БД), $\rho$ - отношение поиска. Таким образом, понятие ЗИП - формализовано.

Следующий шаг - это выбор модели для алгоритма поиска. Рассмотрим произвольную ЗИП $S_{z}=(X, Y, p)$. Считается, что алгоритм поиска решает ЗИП, если на 
любой запрос из множества запросов $X$ он выдает все те и только те записи из $Z$, которые удовлетворяют запросу. Выберем произвольную запись $Z \in Z$. Для нее введем характеристическую функцию

$X_{z, p}(x)=\left\{\begin{array}{c}1, \text { если } x p z \\ 0, \text { в противном случае }\end{array}\right.$,

T.e. функция равна 1 на тех запросах, которым удовлетворяет запись z. Можно утверждать, что алгоритм, решающий ЗИП $S_{z}=(X, Z, p)$, где $Z=\left\{z_{1}, z_{2}, \ldots, z_{k}\right\},-$ ни что иное, как алгоритм, реализующий систему функций $\left\{x_{z 1, p}, x_{z 2, p}, \ldots, x_{z k, p}\right\}$. Следовательно, управляющая система, моделирующая алгоритм, решающий ЗИП $S_{z}=$ $(X, Z, p)$, должна представлять собой многополюсник, реализующий множество характеристических функций $\left\{x_{z 1, p}, x_{z 2, p}, \ldots, x_{z k, p}\right\}$.

Сеть с описанным функционированием в качестве решения поставленной в работе задаче является частным случаем информационного графа.

Таким образом, математическая модель позволяет контролировать, прогнозировать и определять объем перевозок, а информационный граф - оценить сложность информационной системы и ее производительность.

\section{4. Разработка подсистемы контрольных процедур транспортно- экспедиционной деятельности}

Для унификации контрольных процедур в работе определена структура и взаимосвязи элементов, систематизированы задачи технологических процессов. В основе построения системы лежит принцип соответствия каждому технологическому процессу определенного списка контрольных процедур $[8,10,11,15]$.

Предложенная система унифицирована для основных технологических процессов оказания транспортных и экспедиционных услуг. Целесообразно выявление точек наибольших рисков с целью последующей реализации контрольных процедур. Для этого в каждом технологическом процессе определяются подпроцессы или ключевые точки, в которых возникают максимальные риски. Для каждого контракта или заказа формируется индивидуальный перечень необходимых контрольных процедур.

Схема построения системы контрольных процедур представлена на рисунке 4.

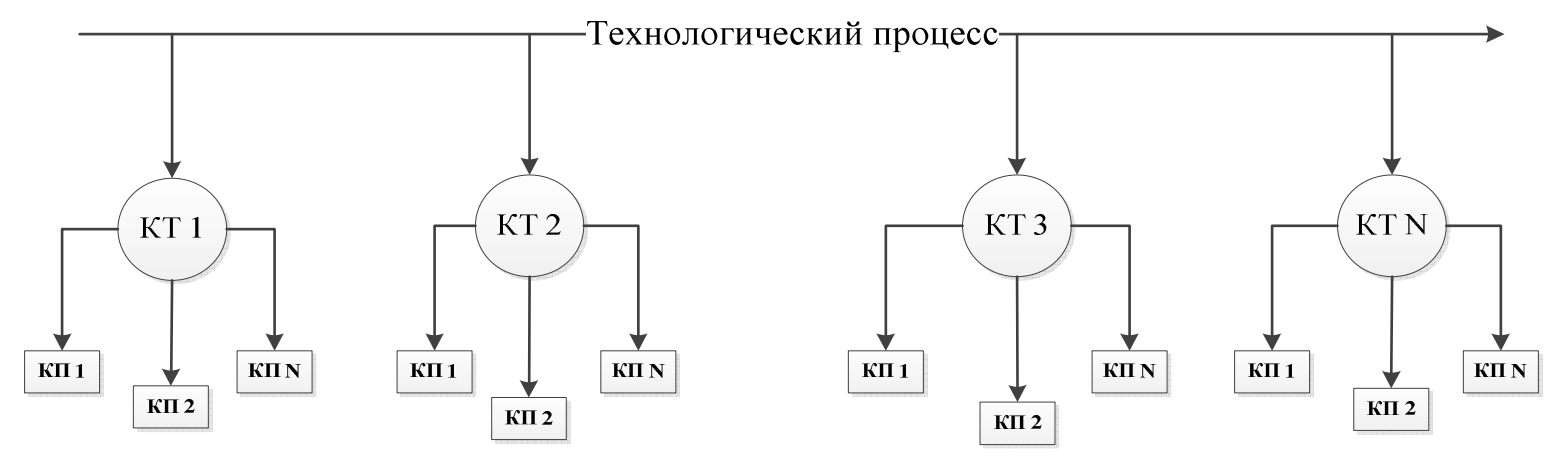

КТ - контрольная точка; КП - контрольная процедура.

Рисунок 4 - Схема построения системы контрольных процедур 
В работе предложен подход к определению контрольных точек для обеспечения непрерывного процесса обработки и сверки данных в целях выявления отклонений между плановыми и фактическими значениями показателей транспортноэкспедиционной деятельности.

Разработанная система контрольных процедур позволяет:

- осуществлять постоянный контроль в режиме реального времени;

- обеспечивать необходимый уровень контроля и выполнение определенных контрольных процедур;

- создавать индивидуальные роли и контролировать цепь подрядчиков;

- создать единую форму отчетов для всех контрагентов;

- конфигурироваться в зависимости от текущих потребностей и будущих интересов, настраиваться под каждый конкретный процесс;

- оперативно устранять или минимизировать последствия возникающих сбоев, выявлять потенциальные проблемы до их возникновения;

- обеспечивать поддержку управленческих решений;

- повышать качество предлагаемых услуг.

Система является унифицированной, масштабной и гибкой, при небольших доработках может быть встроена в любой программный продукт, который используется в транспортно-экспедиционной деятельности.

В работе процесс внедрения блока контроля в систему рассмотрен на примере программно-моделирующего комплекса, используемого на ТЭП. Алгоритм контроля при транспортировке грузов представлен на рисунке 5.

Действующий программно-моделирующий комплекс обеспечивает систему контроля информацией о статусе перевозки в реальном времени и делает возможным выбор формата представления информации. При этом в действующем комплексе отсутствует функция отслеживания фактических значений основных показателей и возможность выбора контролируемой системы параметров.

Учитывая особенности каждой сделки, возникает необходимость измерить, оценить и сравнить результаты на основе показателей всех технологических процессов, а также выявить причины отклонений, если сделка в сопоставимых условиях является более затратной. Информационный поток, связанный с осуществлением контрольных функций при формировании блока контроля предлагается отражать параллельно существующим материальным и информационным потокам, что позволяет в режиме реального времени сопоставлять фактические показатели с расчетными данными и идентифицировать причины отклонений.

Система идентифицирует и оценивает отклонения фактических показателей от плановых. При появлении сбоев, не нарушающих условий контракта, экспертная подсистема автоматически определяет адресаты передачи информации, после чего реализует процедуру устранения инцидентов (рисунок 6). 


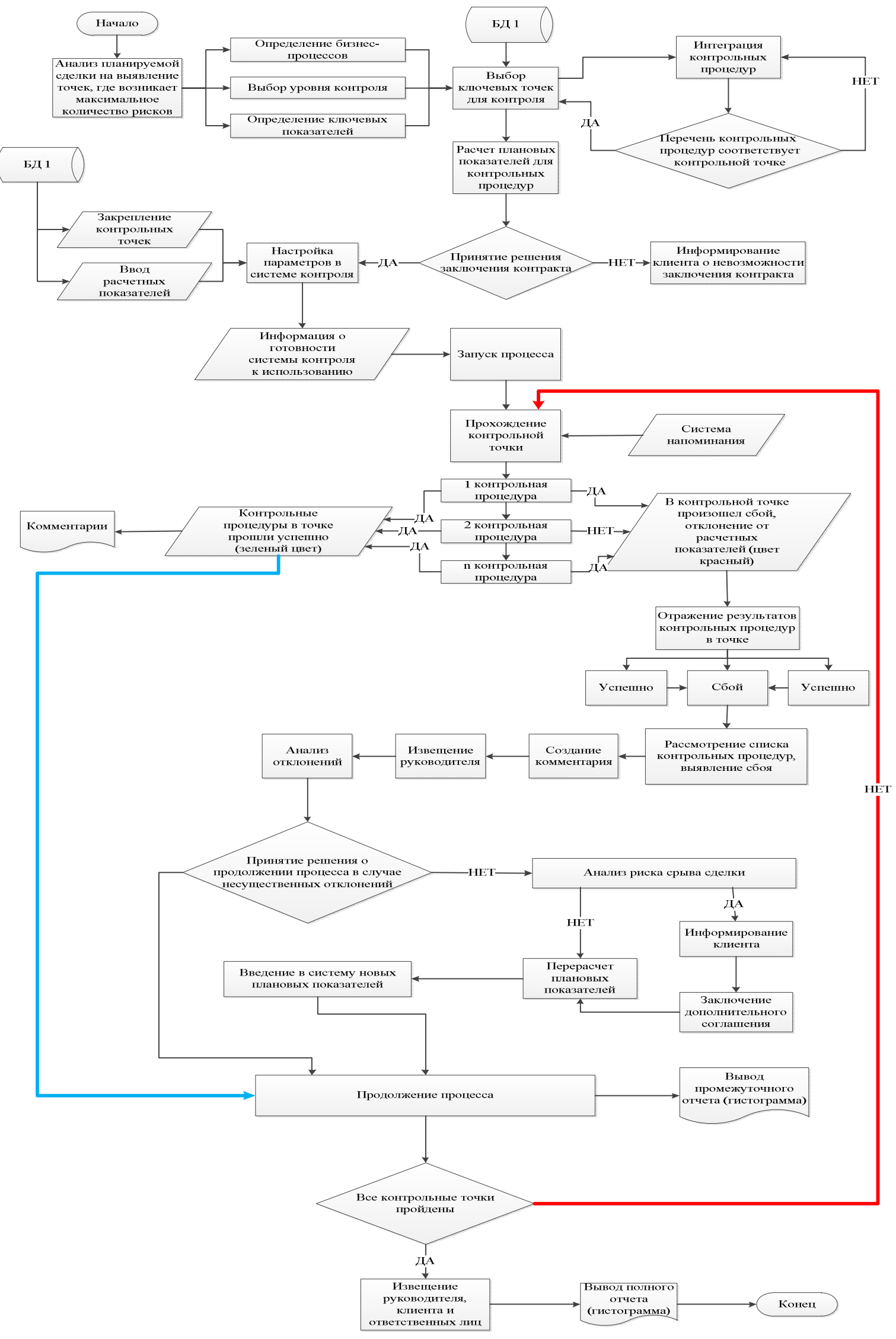

Рисунок 5 - Алгоритм контроля транспортировки грузов 


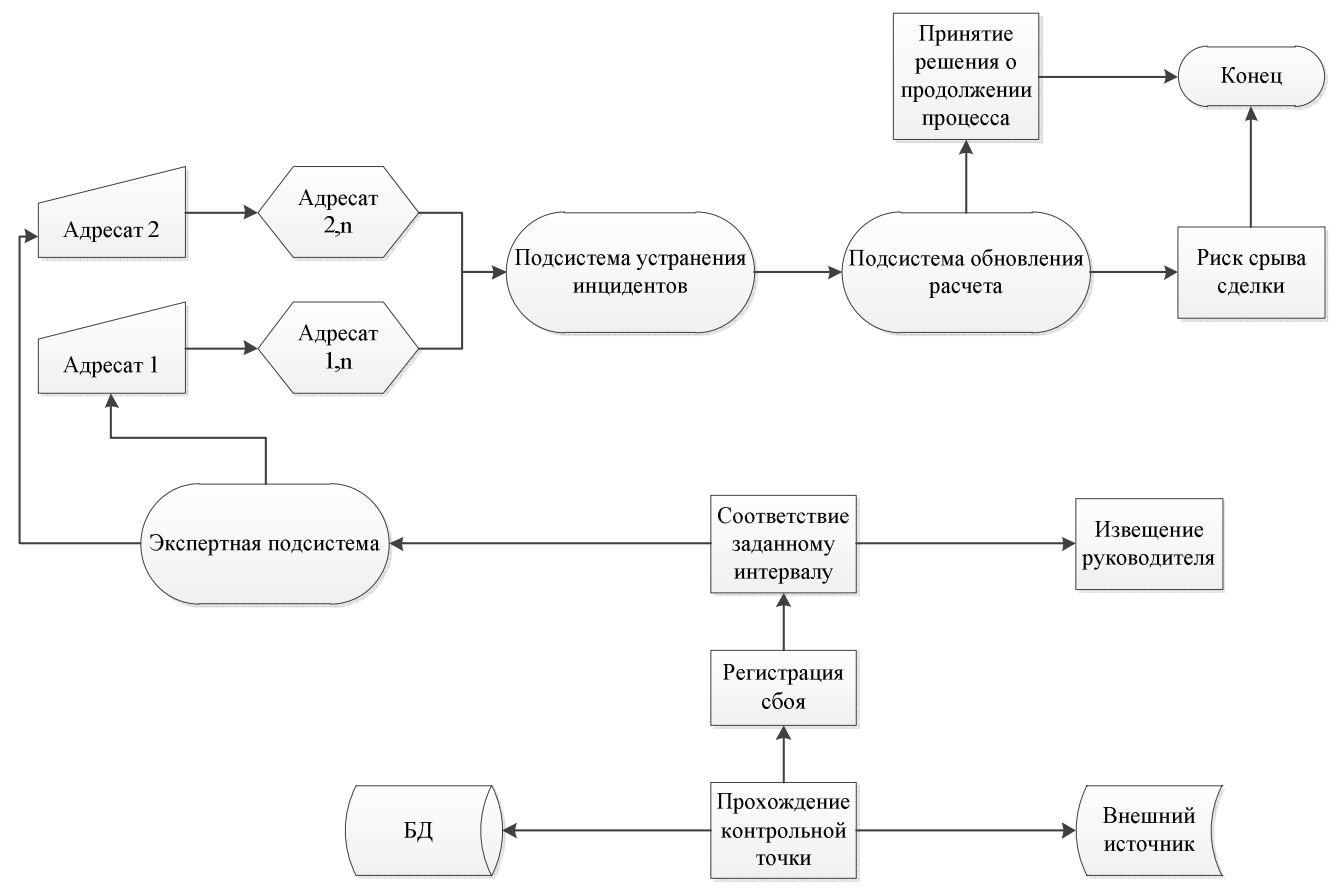

Рисунок 6 - Схема управления устранением инцидентов

При попадании фактических данных в интервал заданных отклонений и, учитывая спланированный на основании предложенной модели спрос, система имеет возможность автоматического перерасчета плановых показателей. Таким образом, последовательно корректируя параметры под оптимальные, реализуется поддержка управленческих решений для последующих сделок (рисунок 7) [10, 12, 13, 23 -27].

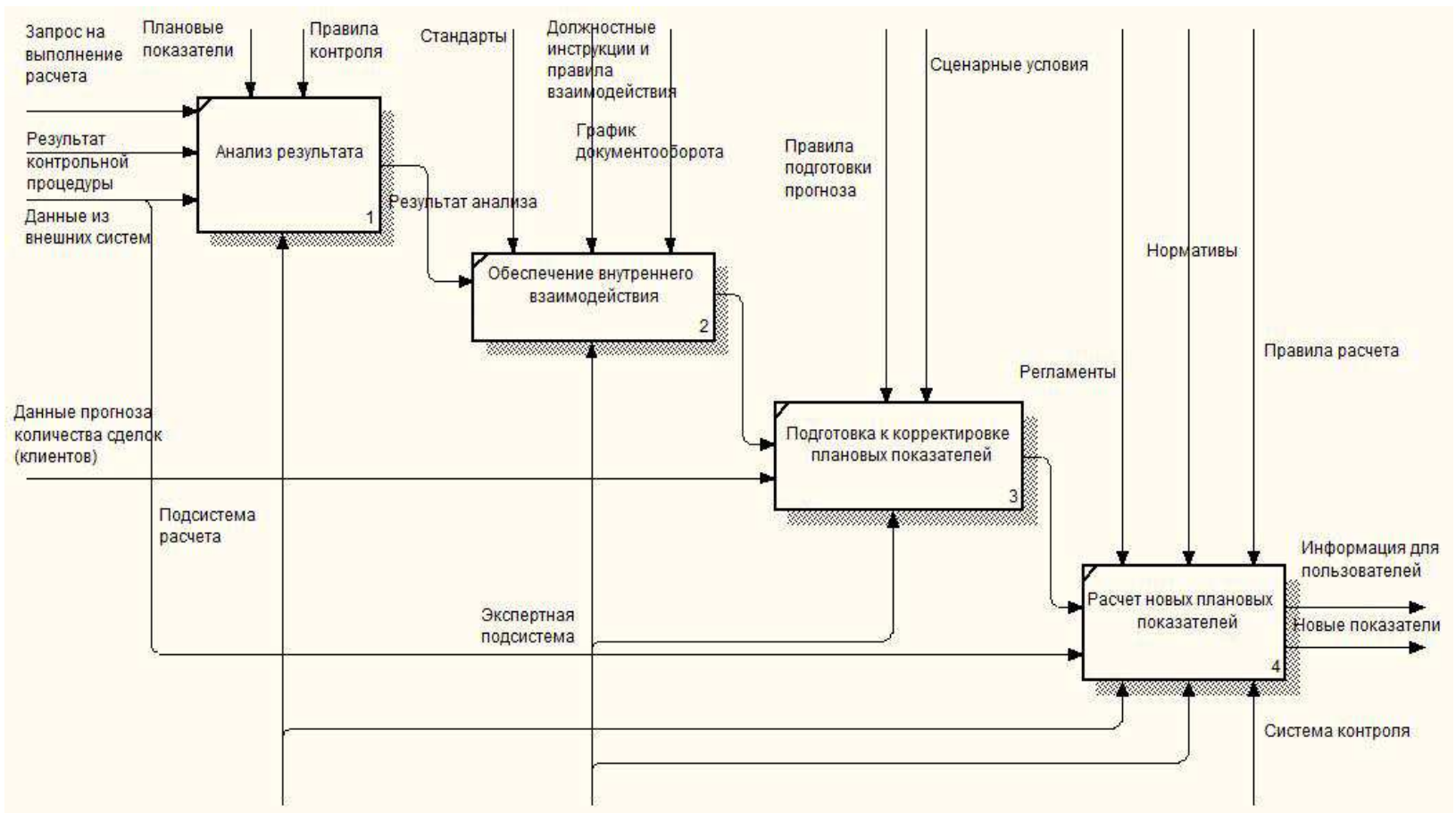

Рисунок 7 - Структурно-функциональная модель системы контроля транспортноэкспедиционного обслуживания 


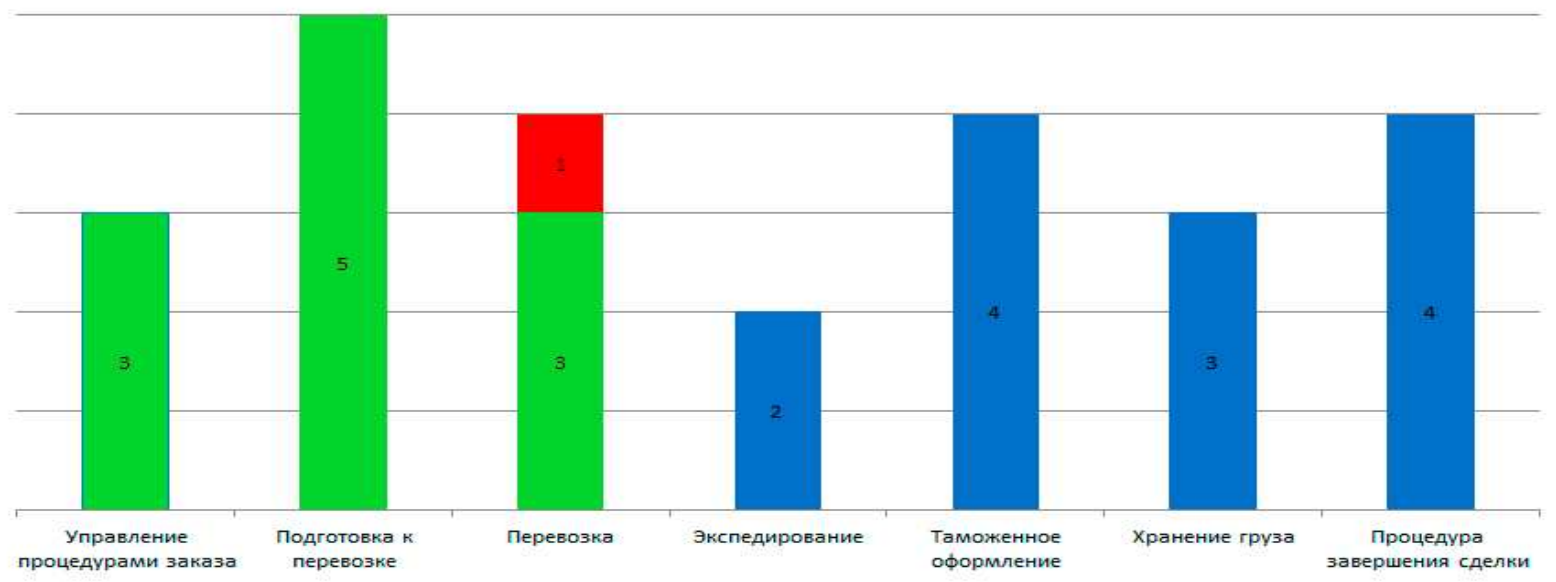

Рисунок 8 - Визуализация результатов

Для наглядного результатов в специально разработанной программе строится диаграмма (рисунок 8), каждый столбец которой описывает технологический процесс и имеет цвет:

- зеленый цвет - фактические показатели технологического процесса соответствуют расчетным;

- желтый цвет - если отклонение показателей от расчетных достаточно велико, но не приводит к нарушению сроков контракта по сделке. В этом случае может возникнуть необходимость в существенном перерасчете плановых показателей, изменении цепи поставки, смене агента.

- красный цвет - если отклонение фактических показателей может привести к срыву обязательств по контракту;

- синий цвет - технологические процессы, срок которых в соответствии с планом проведения перевозки еще не наступил.

Отчет в виде гистограммы позволяет мгновенно оценить результат по сделке, проанализировать своевременность и правильность принятия управленческих решений, а также разработать и осуществить мероприятия, позволяющие минимизировать возникновение подобных проблем в будущем.

Основные преимущества использования системы состоят в том, что она:

- является унифицированной и прозрачной, встраивается в любой программный продукт, предполагает создание ролей для третьих лиц;

- позволяет обеспечивать необходимый уровень контроля в режиме реального времени;

- предполагает единую форму отчетов для всех контрагентов;

- адаптируется к текущим потребностям и будущим интересам бизнеса, легко настраивается под каждую конкретную сделку;

- позволяет выделить ключевую часть процесса, при этом менеджмент тратит меньше времени на незначительные процессы; 
- оперативно выявляет потенциальные проблемы до их возникновения, минимизирует последствия возникающих сбоев.

\section{5. Оценка эффективности проекта по созданию и внедрению системы контроля транспортно-экспедиционной деятельности}

Для оценки используются показатели чистой приведенной стоимости (NPV), внутренней нормы рентабельности (IRR), приведенного срока окупаемости (Pay-back Period), периода окупаемости (T) и рентабельности инвестиций (ROI). Если значение ROI < 20\%, то осуществленные инвестиции в проект неэффективны. «Агрессивные» предприятия должны ориентироваться на ROI около 150-300\%. При этом любое положительное значение NPV считается показателем хорошей эффективности проекта.

Расчет производился на основании статистических данных чешского отделения компании BDP International, где проходило внедрение системы контроля. Ниже, на рисунке 9, представлены экономические показатели деятельности компании в условиях действующего программного продукта и после внедрения интегрируемого модуля контроля.

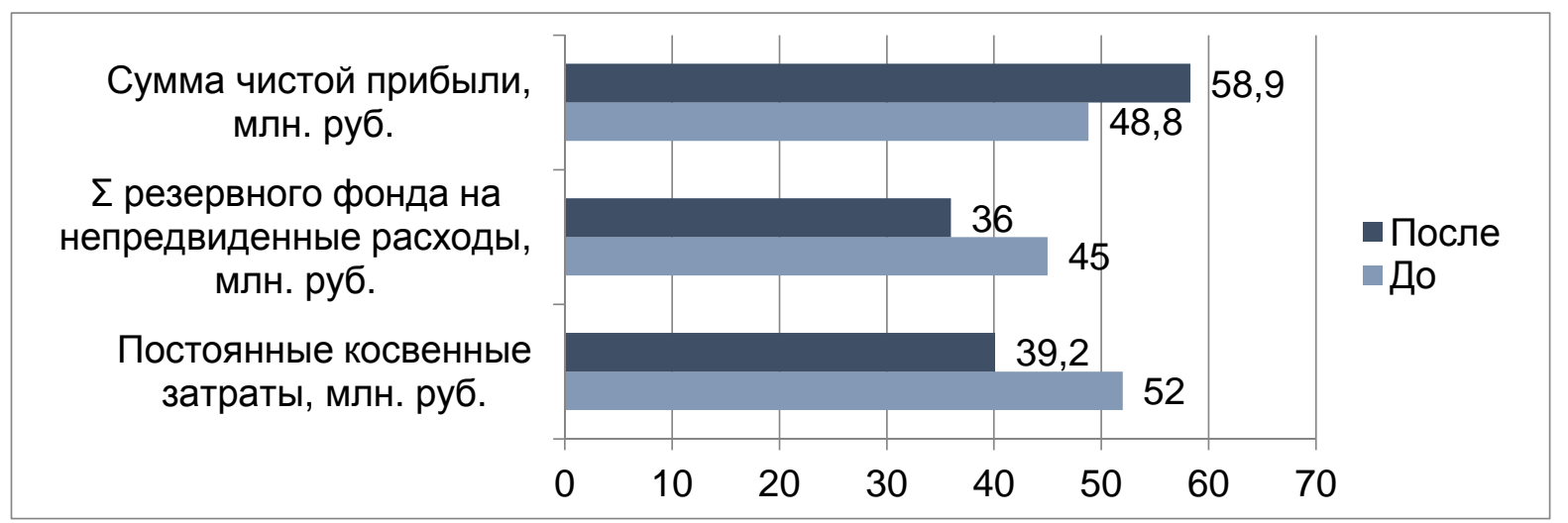

\section{Рисунок 9 - Эффективность использования системы контроля}

Исходя из расчетов, период окупаемости проекта составил около 4 лет. Показатель инвестиционной эффективности проекта ROI составил 207\%, прирост чистой прибыли - 305,4 тыс. долл., чистая приведенная стоимость NPV - 5,26, a рентабельность - 24\%. Результат расчета показал, что данный инвестиционный проект экономически эффективен. Также улучшается качество информации, ее достоверность и точность, сокращается время на обработку, обеспечивается необходимый уровень безопасности, следовательно, повышается уровень управления информацией.

\section{6. Заключение}

Таким образом, проведен анализ современных тенденций развития транспортноэкспедиционных предприятий и систем поддержки управленческих решений. Выявлено, что наибольшее число рисков возникает при осуществлении оперативной деятельности. 
Сравнительный анализ возможностей существующих программных продуктов выявил их эксплуатационные недостатки, что явилось обоснованием необходимости разработки блока контроля.

Разработана математическая модель расчета плановых показателей для целей управления процессами в транспортно-экспедиционной деятельности и реализован метод выбора управленческого решения на основе информационного графа.

Для решения задачи организации системы контроля транспортно-экспедиционной деятельности предлагается использование системы контрольных процедур, которая обеспечивает необходимый уровень контроля в режиме реального времени.

Разработана унифицированная система контрольных процедур и предложен подход к определению ключевых точек для обеспечения непрерывного процесса обработки и сверки данных.

Для обеспечения оптимального достижения целей транспортно-экспедиционной деятельности и снижения неопределенностей и рисков оперативной деятельности разработан алгоритм осуществления процесса контроля транспортных и информационных потоков.

Для поддержки принятия управленческих решений разработана схема управления устранением инцидентов с возможностью автоматического перерасчета плановых показателей.

Разработана методика управления и планирования технологических процессов на транспортно-экспедиционном предприятии. Осуществлена программная реализация предложенных методик и моделей.

Разработанные методики, модели, алгоритмы и программы прошли апробацию и внедрены для практического применения в ряде предприятий.

\section{Список информационных источников}

[1] Крупенский, Н.А. Разработка и внедрение информационной системы нормативного обеспечения бизнеса на автотранспортном предприятии / Н.А. Крупенский // Грузовое и пассажирское автохозяйство. - 2010. - №2 - С. 42-44.

[2] Крупенский, Н.А. Методологии создания ИТ-систем на транспорте / Н.А. Крупенский// Грузовое и пассажирское автохозяйство. - 2010. - №8. - С. 38-44.

[3] Крупенский, Н.А. Информационные системы и их роль в современном ведении бизнеса на транспортном предприятии / Н.А. Крупенский // Вестник Московского Автомобильно-Дорожного Государственного Технического Университета (МАДИ). - 2011. - Вып. 4(27). - С. 59-62.

[4] Крупенский, Н.А. Разработка блока оперативного контроля управления перевозками./ А.М. Ивахненко, Н.А. Крупенский // Вестник Московского Автомобильно-Дорожного Государственного Технического Университета (МАДИ). - 2012. - Вып. 1(28). - С. 123-127.

[5] Крупенский, Н.А. Основные цели бизнес-направления «Нормативное обеспечение бизнеса» / А.М. Ивахненко, Н.А. Крупенский, А.А. Федоренко// Сборник научных трудов студентов и аспирантов факультета «Управление»: Модернизация технологий управления в автотранспортных системах. - М.: Московский 
Автомобильно-Дорожный Государственный Технический Университет (МАДИ), 2010. - С. 224-228.

[6] Крупенский, Н.А. Информационное обеспечение логистической системы «ИС НОБ» / Н.А. Крупенский// Интегрированная логистика. - 2010. - №6. - С. 38-40.

[7] Крупенский, Н.А. Использование контрольных процедур в целях оперативного управления транспортными процессами / Н.А. Крупенский// Сборник статей: 13-й Международный форум «Высокие технологии XXI века». - М.: «ЭКСПО-ЭКОС», 2012. - C. 14-22.

[8] Ивахненко А.М., Крупенский Н.А. Разработка блока контроля в информационной системе управления транспортными потоками // Автоматизация и управление в технических системах. - 2012. - № 1. - С. 135-140.

[9] Куфтинова Н.Г. Процессно-ориентированный подход к автоматизации планирования и управления транспортировкой продукции предприятий промышленности / А.В. Остроух, Н.Г. Куфтинова // Вестник МАДИ - 2010. - Вып. 4(23). - C. 62-66.

[10] Куфтинова Н.Г. Разработка информационно- логической модели транспортной сети мегаполиса / А.В. Остроух, Н.Г. Куфтинова // Бюллетень транспортной информации. - М.: Национальная ассоциация транспортников, 2013. - №1 (211). C. 23-26.

[11] Остроух А.В. Оперативный контроль транспортно-экспедиционной деятельности. Процессный подход к агрегированию системы показателей деятельности транспортно-экспедиционного предприятия / А.В. Остроух, А.М. Ивахненко, Н.А. Крупенский. - Saarbrucken, Germany: Palmarium Academic Publishing, 2013. - 221 p. - ISBN 978-3-659-98329-0.

[12] Николаев А.Б. Информационные технологии в менеджменте и транспортной логистике: учебное пособие / А.Б. Николаев, А.В. Остроух. - Saint-Louis, MO, USA: Publishing House Science and Innovation Center, 2013. - 254 c. - ISBN 978-0615-67110-9.

[13] Суркова Н.Е. Методы проектирования информационных систем / А.В. Остроух, Н.Е. Суркова. - М.: РосHОУ, 2004. - 144 с. - ISBN 5-89789-021-8.

[14] Остроух А.В., Башмаков И.А. Минимизация производственных рисков при автотранспортном обслуживании потребителей бетонных смесей // Автоматизация и управление в технических системах. - 2013. - № 4.2. - С. 83-90. DOI: $10.12731 / 2306-1561-2013-4-40$.

[15] Порфирьева С.А. Автоматизированные информационные системы на автотранспортном предприятии / А.В. Остроух, К.А. Данчук, А.Б. Львова, С.А. Порфирьева, П.С. Якунин // В мире научных открытий. Серия «Проблемы науки и образования». - 2012. - №2.6 (26). - С.34-38.

[16] Остроух А.В., Башмаков И.А. Процессная модель технологии транспортировки бетонных смесей автомобильным транспортом // Автоматизация и управление в технических системах. - 2013. - № 4.1. - С. 75-81. DOI: 10.12731/2306-1561-20134-14.

[17] Остроух А.В., Джха Прабхакар. Автоматизированная система управления складом железобетонных изделий // Автоматизация и управление в технических системах. - 2013. - № 4.1. - С. 51-56. DOI: 10.12731/2306-1561-2013-4-9.

[18] Остроух А.В., Синха Бабу Раджа. Исследование информационных систем управления взаимоотношениями с поставщиками // Автоматизация и управление в технических системах. - 2013. - № 4.1. - C. 56-62. DOI: 10.12731/2306-15612013-4-10. 
[19] Остроух А.В., Джха Прабхакар, Башмаков И.А., Польгун М.Б. Обзор технологий транспортировки бетонных смесей автомобильным транспортом // Автоматизация и управление в технических системах. - 2013. - № 4.2. - C. 178-189. DOI: 10.12731/2306-1561-2013-4-38.

[20] Остроух А.В., Башмаков И.А., Польгун М.Б. Оптимизация параметров процессов автотранспортного обслуживания потребителей бетонных смесей // Автоматизация и управление в технических системах. - 2013. - № 4.2. - С. 189198. DOI: $10.12731 / 2306-1561-2013-4-39$.

[21] Остроух А.В., Башмаков И.А. Минимизация производственных рисков при автотранспортном обслуживании потребителей бетонных смесей // Автоматизация и управление в технических системах. - 2013. - № 4.2. - С. 83-90. DOI: 10.12731/2306-1561-2013-4-40.

[22] Польгун М.Б. Применение графов дорожной сети в автоматизированной системе управления транспортировкой дорожно-строительных материалов / А.В. Остроух, М.Б. Польгун, В.В. Тихоцкий // Приборы и системы. Управление, контроль, диагностика. - М.: «Научтехлитиздат», 2014. - №1. - С. 12-17.

[23] Помазанов А.В. Методика оптимизации баз данных / А.В. Остроух, А.В. Помазанов, А.И. Белоусова, А.О. Васильева // В мире научных открытий. Серия «Проблемы науки и образования». - 2012. - №12. - С.49-54.

[24] Ostroukh A.V., Kuftinova N.G. Automation of Planning and Management of the Transportation of Production for Food Processing Industry Enterprises // Automatic Control and Computer Sciences. - 2012. - Vol. 46. - No. 1. - pp. 41 - 48. DOI: 10.3103/S0146411612010063.

[25] A.V. Ostroukh, I.A. Bashmakov, N.E. Surkova. Process Model of the Technology of Concrete Mixtures Transportation by Road // World Applied Sciences Journal (WASJ). 2014. Vol. 31, No 4. pp. 500-507. DOI: 10.5829/idosi.wasj.2014.31.04.333.

[26] A. Ostroukh, A. Pomazanov. Realtime Development and Testing of Distributed Data Processing System for Industrial Company // Middle-East Journal of Scientific Research. 2014. Vol. 20 (12). pp. 2184-2193. DOI: 10.5829/idosi.mejsr.2014.20.12.21106.

[27] Ostroukh A.V., Belousova A.I., Pavlov D.A., Yurchik P.F. Problems of organization and search the knowledge base in the CRM-systems // IOSR Journal of Engineering (IOSRJEN). 2014. Vol. 04. Issue 02. V3. pp. 18-23. DOI: 10.9790/3021-04231823. ANED: 0.4/3021-04231823. 